\title{
Dynamic Bandwidth Allocation Algorithm Based on Two-Phase Cycle for Efficient Channel Utilization on Ethernet $\mathrm{PON}^{\star}$
}

\author{
Won Jin Yoon, Woo Jin Jung, Tae-Jin Lee, Hyunseung Choo, \\ and Min Young Chung ${ }^{\star \star}$ \\ School of Information and Communication Engineering \\ Sungkyunkwan University \\ 300, Chunchun-dong,Jangan-gu, Suwon, Kyunggi-do, 440-746, Korea \\ $+82-31-290-7990$ \\ \{yoon1007,jwj0107,tjlee, choo,mychung\}@ece.skku.ac.kr
}

\begin{abstract}
Ethernet passive optical network (EPON) is a low-cost and high speed solution to the bottleneck problem. We study previous interoptical network unit (ONU) scheduling algorithm, interleaved polling with stop (IPS).The IPS algorithm needs an idle time period between two consecutive frame transmission cycles on uplink. In this paper, we propose a two-phase cycle dynamic bandwidth allocation (TCDBA) algorithm to increase the channel utilization on uplink through the elimination of the idle period. And, we also evaluate the performance of the proposed algorithm by simulations and confirm its effectiveness.
\end{abstract}

Keywords: Ethernet passive optical network (EPON), multipoint control protocol (MPCP), dynamic bandwidth allocation (DBA).

\section{Introduction}

In order to adapt data traffic increasing due to the advent of various multimedia applications, EPON has been introduced 1] 2. In EPON, all data to be transmitted are encapsulated in Ethernet frames. Thus, worldwide-spread Ethernetbased LANs can be cost-effectively interconnected through EPON. An EPON is a point-to-multipoint optical network consisting of one optical line terminator (OLT), several ONUs, and a passive optical splitter/combiner [1]. The EPON uses multipoint control protocol (MPCP) to exchange control messages between an OLT and ONUs for the time slot request and allocation 2].

Inter-ONU scheduling algorithms are responsible for arbitrating the transmissions of ONUs. However, the EPON dose not establish standardization of data

\footnotetext{
* This work was supported by the Korea Research Foundation Grant funded by the Korean Government(MOEHRD)(KRF-2005-042-D00248) and by the MIC(Ministry of Information and Communication), Korea, under the ITRC(Information Technology Research Center) support program supervised by the IITA(Institute of Information Technology Assessment), IITA-2006-(C1090-0603-0046).

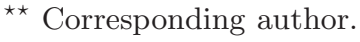


transmission scheduling algorithm between ONUs. In order to fairly distribute uplink resource to all ONUs, the IPS algorithm has been proposed 3 . In the IPS algorithm, an OLT allocates the time duration based on time slot requests of entire ONUs. However, the IPS needs the computation time to calculate time durations to be assigned to the entire ONUs. During the computation time, any ONU can not transmit data to the OLT. In order to use this waste time duration, Assi et al. proposed fast gate DBA (FGDBA) 4. However, in FGDBA, if the request volume of all the ONUs is larger than the predetermined threshold value, the operation of the FGDBA is similar to that of the IPS. Hence, it can not efficiently use the waste time duration under heavy traffic loads.

In this paper, in order to fully utilize uplink in EPON, we propose a TCDBA algorithm. In TCDBA, each cycle is divided into two phases. During the first phase, OLT can receive data from all ONUs while computing the available time durations to be allocated in the second phase.

\section{TCDBA (Two-Phase Cycle Dynamic Bandwidth Allocation) Algorithm}

In TCDBA, a transmission cycle is divided into two phases, phases 1 and 2, in order to efficient use uplink. The time duration of phase 1 is set as the fixed value which is larger than the sum of DBA computation time and the maximum round trip time (RTT), $T_{m r t t}$, between the OLT and ONUs. However, the time duration of phase 2 is varied as the request information of all the ONUs. OLT can inform all the ONUs of their available time durations before the corresponding ONUs start their data transmissions on uplink in the phase 2. Hence, the phase 2 starts after the instant of ending phase 1.

Let $T_{\text {phase } 1}$ be the sum of the DBA computation time, $T_{\text {comp }}$, and $T_{m r t t}$. and it is equally divided into $N$ time slots, $A^{p 1}$. As soon as the OLT receives a request volume $V_{i}$ (bytes) from ONU $i(i=1, \ldots, N)$, the OLT determines the remaining request volume, $V_{i}^{p 2}$, for $\mathrm{ONU} i$. $V_{i}^{p 2}$ is calculated as $\max \left(0, V_{i}-A^{p 1}\right)$. After the OLT informs all the $N$ ONUs of $A^{p 1}$, it starts DBA computation based on $V_{i}^{p 2}$ for all $i(i=1, \ldots, N)$ to allocate the available time slots in the upcoming phase 2 . To compute the available time slots, we first decide the length of the current transmission cycle, $T_{\text {cycle }}$, as follows.

$$
T_{\text {cycle }}=\min \left(T_{\max }, \sum_{i}^{N}\left(2 \cdot T_{g}+\frac{V_{i} \times 8}{C}\right)\right),
$$

where $T_{g}$ is a guard time interval between the two consecutive time durations, $T_{\max }$ is the length of the maximum-permitted transmission cycle and $C$ is the link capacity between the OLT and all the ONUs. Thus, the time duration of phase $2, T_{\text {phase } 2}$, becomes the difference between $T_{\text {cycle }}$ and $T_{\text {phase } 1}$. For each ONU, the size of the minimum guaranteed time slot, $B_{\min }$, in the upcoming phase 2 is given by $\left(T_{\text {phase } 2} / N\right)-T_{g}$. Based on $B_{\text {min }}$ and $V_{i}^{p 2}$, the OLT classified all the ONUs into two subset, $M$ and $M^{c}$. $M$ is the set of ONUs with $V_{i}^{p 2} \leq B_{\text {min }}$ 


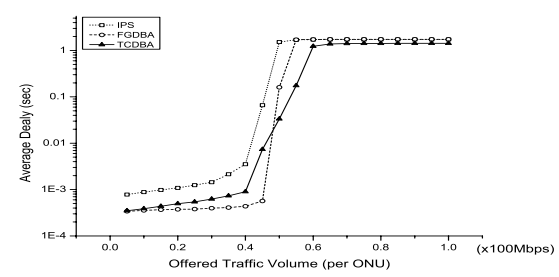

(a) Average packet transmission delay for IPS /FGDBA/TCDBA

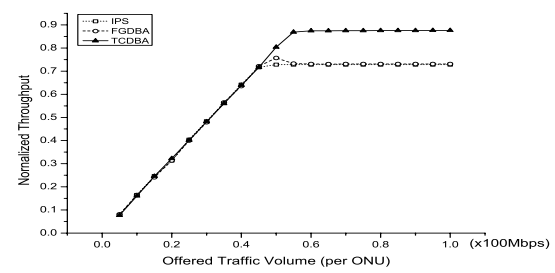

(b) Normalized throughput for IPS/FGDBA /TCDBA

Fig. 1. Simulation results for IPS /FGDBA/TCDBA

and $M^{c}$ is the complement of $M$. For ONU $i$ in $M$, the OLT allocates time slot, $A_{i}^{p 2}$, in the phase $2 . A_{i}^{p 2}$ is the smaller one between $B_{\min }$ and $V_{i}^{p 2}$. To allocate the remaining time to all the ONUs in $M^{c}$, the OLT calculates the total excessive time $\left(B_{\text {total }}^{\text {excess }}=\sum_{i \in M}\left(B_{\text {min }}-V_{i}^{p 2}\right)\right)$. For ONU $j$ in $M^{c}$, the excessive time, $B_{j}^{\text {excess }}$, is given by

$$
B_{j}^{\text {excess }}=\frac{B_{\text {total }}^{\text {excess }} \times V_{j}^{p 2}}{\sum_{j \in M^{c}} V_{j}^{p 2}} .
$$

Finally, the OLT allocates the time slot used in phase 2 to ONU $j$ in $M^{c}$ as follows.

$$
A_{j}^{p 2}=B_{\min }+B_{j}^{\text {excess }} .
$$

\section{Performance Evaluation}

We use an EPON architecture with 16 ONUs and an OLT that expends $200 \mu \mathrm{s}$ for the DBA computation. The link capacity between an OLT and ONUs is $1 \mathrm{Gbps}$. The maximum cycle time is set to $2 \mathrm{~ms}$ and each ONU has a $10 \mathrm{Mbytes}$ buffer. The guard time between two consecutive time slots is set to $1 \mu \mathrm{s}$. The inter-frame gap between two Ethernet frames is 12 bytes. We, also, assume that the round trip time between the OLT and all the ONUs is the same as $200 \mu \mathrm{s}$. Each end-user generates high class packets with 70 bytes as a Poisson process, and medium/low class packets as a self-similar traffic pattern [5]. 
The average delay of IPS, FGDBA, and TCDBA varying offered traffic volume $\rho$ per ONU is shown in Fig. 1(a). The average packet transmission delay of the TCDBA is always less than that of the IPS, because the IPS can not use the uplink during the sum of $T_{\text {comp }}$ and $T_{m r t t}$. Since the TCDBA can fully use the idle time, it transmits more upstream data traffic than other algorithms. Especially, for $45 \mathrm{Mbps} \leq \rho$, its delay is smaller than that of FGDBA and IPS. Moreover, for $\rho$ in [40Mbps, 60Mbps] the average delay of FGDBA drastically increases as $\rho$ increases. The reason is that some ONUs can not transmit their upstream data during idle time, because their request volume is larger than the predetermined threshold value. The waste of this period in FGDBA will make the increase of the average packet transmission delay. However, in the TCDBA, since the change of the traffic load dose not influence on the use of idle time, the average transmission delay more slowly increases. Fig. 1(b)] shows the normalized throughput of IPS, FGDBA and TCDBA. Compared with the IPS and FGDBA, the proposed TCDBA, the maximum throughputs are about $71.5 \%, 75 \%$, and $87.4 \%$, respectively. Under overload condition, i.e., $60 \mathrm{Mbps}<\rho$, the maximum throughput of TCDBA is improved about $15.9 \%$ compared with that FGDBA.

\section{Conclusion}

This work proposed the TCDBA algorithm in order to fully use the uplink and improve the throughput. From the simulation results, TCDBA shows a higher performance than other algorithms in terms of low delay and high throughput. For further studies, the performance evaluation of the TCDBA algorithm is required for supporting differentiated services.

\section{References}

1. M. P. McGarry, M. Maier, and M. Reisslein : Ethernet PONs: A Survey of Dynamic Bandwidth Allocation (DBA) Algorithms. IEEE Journal of Lightwave Tech. Vol. 22 No. 11 (2004) 2483-2497

2. IEEE 802.3 Ethernet in the First Mile Study Group[Online]. Available: http://www.ieee802.org/3/efm/public/index.html

3. J. Xie, S. Jiang, and Y. Jiang : A Dynamic Bandwidth Allocation Scheme for Differentiated Services in EPONs, IEEE Optical Comm. Vol. 42 Issue 8 (2004) pp. S32-S39

4. C. M. Assi, Y. YE, S. Dixit, and M. A. Ali : Dynamic Bandwidth Allocation for Quality-of-Service Over Ethernet PONs, IEEE Journal on Sel. Areas in Comm. Vol. 21 No. 9 (2003) pp. 1467-1477

5. D. Sala and A. Gummalla : PON Functional Requirements: Services and Performance [Online]. Available: http://grouper.iee.org/groups/802/3/efm/public/ jul01/presentations/sala_1_0701.pdf 